\title{
El cuento como estrategia didáctica para fomentar la habilidad escrita
}

Sara Inés Montaña Cárdenas*

Artículo de reflexión

Fecha de Recepción: 3 diciembre 2017.

Fecha de Aprobación: 18 abril 2018.

\section{Resumen}

El documento de reflexión evidencia la necesidad de incorporar una estrategia didáctica que fomente la habilidad escrita. El interés de abordar esta problemática se soportó en la pregunta ¿cómo fortalecer la habilidad escrita de los estudiantes de grado sexto?; estuvo dirigido al observar las dificultades en la construcción de textos escritos entre las que se encuentran: falta de vocabulario que dé a conocer de manera clara y coherente lo que se quiere decir. La metodología hizo uso del enfoque cualitativo, y mediante la investigación acción educativa se llevó a cabo el desarrollo de la misma; se realizó un diagnóstico empleando un instrumento que permitió determinar las fortalezas y debilidades con respecto a la habilidad escrita. Participaron 15 docentes, 28 padres de familia y 28 estudiantes de grado sexto de básica secundaria de una institución educativa del municipio de Paipa (Boyacá). El análisis de información se realizó a partir de la triangulación de datos. Los resultados mostraron que la escuela debe propiciar la habilidad en producción de textos, porque favorece la adquisición de conocimientos, mediante el cuento como estrategia didáctica, aun cuando existen diversidad de textos que se pueden trabajar con los estudiantes sobre la práctica de la escritura, se pudo apreciar que para los niños este tipo de narrativas tiene gran aceptación.

\section{Palabras clave}

Fortaleza, Dificultad, Habilidad Escrita, El Cuento, Estrategia Didáctica
* Institución Educativa

Armando Solano saraimontana@gmail.com

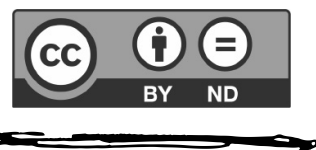




\section{Introducción}

La comunicación a lo largo de la historia ha sido una característica propia del hombre, la cual lo diferencia de otras especies y ha consolidado el raciocinio como elemento transformador y dinamizador de las actividades propias y evolutivas de los seres humanos. Por tal razón se piensa que dicha competencia ha permitido la conservación, acumulación y transmisión de gran cantidad de conocimientos descubiertos por estudiosos y científicos a través del tiempo. Estos conocimientos no hubieran llegado al ser humano si no existiera la escritura, que se presta, no solo para conocer la historia, sino para entretenerse con múltiples obras, que gracias a su excelente construcción han influido e inspirado a muchos para seguir aportando al conocimiento y cultura en distintas épocas y rincones del mundo. En este sentido se han realizado variados estudios que dan cuenta del desarrollo de la habilidad escrita, trabajos que esta investigación tendrá en cuenta como antecedentes a nivel internacional, entre ellos se tienen:

Bazán, Urbina, Domínguez, \& Mansillas (2011), en el trabajo "Desarrollo funcional de competencias de producción de textos en alumnos con bajo desempeño en el último año de primaria", aporta al trabajo que se adelanta importantes fundamentos teóricos y otra manera de abordar la producción de la escritura desde el aula de clase.

En el mismo sentido un trabajo atrayente en relación con la producción de escritura, se titula: "Escritura colaborativa en alumnos de primaria: un modelo social de aprender juntos" elaborada por Gúzman \& Rojas (2012). Este tipo de experiencia aportan a la investigación en la medida que muestra que la práctica de la escritura desde la escuela fortalece la producción textual, desarrollando además otro tipo de habilidades cognitivas y sociales que los estudiantes pondrán en práctica tanto en la vida académica, como en su entorno social de manera exitosa. Finalmente, Álvarez \& Ramírez (2006), en el trabajo "Teorías o modelos de producción de textos en la enseñanza y el aprendizaje de la escritura", resulta de gran importancia para la investigación puesto que deja ver de manera clara una concepción sobre la escritura, su influencia en los procesos cognitivos y plantea como finalidad fomentar la competencia escrita en situaciones tanto académicas como sociales.

En el ámbito nacional se encuentran trabajos como el propuesto por Barletta, Toloza, Del Villar, Rodríguez \& Bovea (2013) titulado "Enseñanza y aprendizaje de la lectura y la escritura". Este trabajo da cuenta en la investigación que se adelanta, que en la labor de enseñanza hay situaciones que escapan a las manos de los docentes y que en muchas ocasiones entorpecen o hacen más lentos los procesos, por tanto, en todo momento el docente debe reflexionar sobre su labor al interior de aula de clase diversificando, actualizando y adaptando sus formas de enseñar. La producción escrita es tan versátil que en ocasiones debe ser combinada con las nuevas tecnologías como lo hicieron 
Henao, Ramírez \& Mejía (2009) con el trabajo "La producción escrita mediada por un programa que reconoce la voz en una experiencia con estudiantes de sexto grado". Se puede decir que este trabajo muestra que, para abordar la escritura desde las aulas, también se puede acudir a las nuevas tecnologías.

En el contexto local se encuentran estudios, como el realizado por Alba \& González (2015) quienes presentan una propuesta teórico - práctica que pretende posibilitar la cualificación del proceso argumentativo y escritural con el propósito de orientar a los estudiantes a desarrollar su pensamiento a través de la producción de nuevas redes de conocimiento, estructurando sus esquemas cognitivos con la construcción de nuevos saberes. Hernández (2008) en su propuesta investigativa, busca fortalecer el proceso sobre las habilidades lingüísticas básicas, a partir de la construcción de significados y sentidos en los actos de conmemoración, resaltando la importancia de promover la escritura desde los primeros años del bachillerato.

Los trabajos descritos anteriormente aportan al estudio patrones académicos del trabajo de aula en el que se desarrollan las habilidades lingüísticas, especialmente sobre escritura en estudiantes, reforzando la necesidad que tiene la educación colombiana de proveer de espacios, en las escuelas, donde los niños y jóvenes puedan desarrollar y manifestar en forma escrita que se están preparando para tareas complejas en la vida académica o cotidiana, haciéndolo con un alto nivel de competencia en lo escrito y como producto de estudios rigurosos que no solo tienen en cuenta al estudiante como sujeto de estudio, sino como ser social que evoluciona y cambia.

En este orden de ideas, las instituciones educativas se preocupan porque los jóvenes adquieran competencias comunicativas que les permita tener acceso a gran cantidad de información, según lo requieran en cualquier momento de su vida. Por tal razón, la lectura y la escritura son instrumentos que orientan a los jóvenes hacia la adquisición del conocimiento con ayuda y orientación de sus maestros desde las escuelas.

Los niños y jóvenes se ven agobiados cuando de plasmar sus ideas se trata, ya sea porque no poseen un vocabulario amplio y adecuado para poder redactar o sencillamente no saben por dónde empezar cuando escriben. Esta es una de las razones por la que los estudiantes, en muchas oportunidades, no obtienen buenos resultados en pruebas externas, ya que su desarrollo implica no solo saber decodificar símbolos (leer en su concepto más básico), sino entender, para poder emitir o transmitir un concepto escrito sobre el tema que se les presenta; para lograrlo el joven debe haber agotado todo un proceso que exige un grado de complejidad importante en la comunicación.

En los estudiantes se vienen observando dificultades en la construcción de textos escritos, entre las que se encuentran: falta de vocabulario amplio y adecuado que permita dar a conocer de manera clara y coherente lo que se quiere decir, 
gran tendencia a copiar literalmente las ideas del texto, dificultad para iniciar la escritura, falta de orden al presentar las ideas o la no inclusión de ideas relevantes que den forma al texto, la falta de concluir o cerrar una idea expresada dejándola inconclusa, rechazo a la mayoría de actividades que involucran la construcción de textos escritos debido a la falta de pericia en esta tarea. Surge de esta manera la pregunta ¿Cómo fortalecer la habilidad escrita de los estudiantes de grado sexto de una Institución Educativa?

Una vez realizado el diagnóstico para determinar las fortalezas y debilidades con respecto a las actividades de escritura de los estudiantes y con el propósito de diseñar e implementar acciones que contrarresten la problemática identificada, este estudio propone fomentar el desarrollo de la habilidad escrita en estudiantes, mediante la utilización del cuento como estrategia didáctica. Aun cuando existe diversidad de textos en los que se pudiera trabajar con los estudiantes sobre la práctica de la escritura, se pudo apreciar que para los niños este tipo de narrativas tiene gran aceptación, haciendo más agradable las actividades que se plantean al producir un texto escrito.

\section{Sustentos Teóricos}

Es innegable la importancia del lenguaje para la formación del individuo y la constitución de la sociedad, ya que se configura como una de las capacidades que ha marcado el curso evolutivo de la especie humana. Esta habilidad ha ayudado a comprender el mundo, a convivir con otros semejantes, a expresar sentimientos en diferentes momentos según los seres humanos lo requieran. El lenguaje se convierte en una herramienta cognitiva que permite tomar conciencia de sí mismo en relación con el entorno social y espacial. Es aquí donde el lenguaje adquiere un doble propósito: uno, de construir seres humanos conscientes de su propio ser, $\mathrm{y}$ otro que está relacionado con lo social, a fin de lograr un crecimiento integral y cooperativo en la apropiación de habilidades que posibiliten compartir expectativas, deseos, creencias, conocimientos, que permitan la difusión y permanente transformación.

En ese sentido Álvarez (2010) establece "la íntima relación que existe entre lenguaje y pensamiento, entre la sicología cognitiva y el interaccionismo social"; en el mismo sentido lo hacen Vigotsky (1985), Habermans (1989), Bruner (1987), Ricoeur (2004) Holliday (2005) y Bronckart (1985), entre otros, conciben el lenguaje como una actividad discursiva que va indisolublemente unida a la actividad humana de la que constituye su fiel reflejo.

En otras palabras, el lenguaje es una característica propia de la actividad social humana, que con el paso del tiempo han ido consolidando formas complejas y funcionales en la actividad discursiva manifestadas en el texto y los discursos de distinta índole. Es así como para Álvarez (2010), la producción y la comprensión de textos han acompañado a la humanidad en los distintos momentos de la evolución cognitiva del ser humano, haciéndolo consiente 
del mundo y del lenguaje ya que "la comprensión y la producción de textos escritos es uno de los fundamentos de la cultura y de la instrucción” (p 45).

En lo que se refiere al cuento como estrategia didáctica, este estimula no solo la lectura sino, también el desarrollo del lenguaje y la creación literaria través de la imaginación. A la vez, permite experimentar de alguna manera situaciones que los personajes de este tipo de textos viven en sus historias. Es innegable que el cuento encuentra gran aceptación entre los niños y jóvenes porque les permite entrar en mundos fantásticos y llenos de posibilidades.

El cuento es un género narrativo de difícil definición por su semejanza con la fábula y la novela corta, especialmente. Meneses (1955) y Bettelheim (1988) están de acuerdo en que la relación oral o escrita de un suceso ficticio parte de la imaginación creadora y no de la fantasía, y se caracteriza por la brevedad, porque se inspira en los problemas propios de los seres humanos.

Como texto narrativo, el cuento se conforma por una trama de acciones, un tiempo donde se ubican las acciones, un tiempo en el que ocurren los hechos, un escenario y unos personajes que dan vida a esas historias fantásticas. Tradicionalmente la estructura del cuento está constituida por un inicio, un nudo y el desenlace.

En la misma óptica Van Dijk (1983) explica que la superestructura del cuento incluye la introducción, compilación, resolución y finalización. Otros autores como Contursi \& Ferro
(2006) interpretan la estructura del cuento de una manera más compleja que comprende una situación inicial, compilación, reacción, resolución, situación final y moraleja. En la actualidad, la composición textual en el cuento rompe muchas veces con estas estructuras queriendo dar un poco más de libertad a los escritores actuales que quieren sorprender con nuevas propuestas, y es que de eso se trata: de liberar la imaginación en busca de nuevas posibilidades en cuanto a la comunicación en lo textual y escrito.

El cuento como texto narrativo se refiere ante todo a acciones de personas, de manera que las descripciones de circunstancia, objetos u otros sucesos quedan subordinadas. Así para Contursi \& Ferro (2006) esta característica semántica se combina con otra de tipo pragmático, en general un hablante explicará unos sucesos $u$ acciones que en cierto modo resulten interesantes.

\section{Metodología}

El desarrollo de este trabajo se enmarca en la investigación acción educativa, desde el enfoque cualitativo. Según Elliot (2000) el propósito de este tipo investigación consiste en profundizar la comprensión del docente sobre una problemática propia de la escuela donde labora, adoptando una postura exploratoria y proponiendo estrategias que den solución a estas situaciones propias del entorno escolar y del aula (Orrego \& Toro, 2014).

Esta investigación se realiza en el municipio de Paipa, en una Institución Educativa con estudiantes de grado
Van Dijk (1983) explica que la superestructura del cuento incluye la introducción, compilación, resolución y finalización. 
sexto. El estudio surgió a partir de las dificultades que evidenciaron los estudiantes en los resultados de las pruebas externas y las actividades académicas.

Para el desarrollo de la investigación y teniendo en cuenta el modelo que desarrolla Elliot (2000) mencionado por Bisquerra (2014) el proceso comprende los siguientes tres momentos:

- La identificación, descripción e interpretación del problema a investigar. Se aplicó un instrumento en el que el estudiante evidenció fortalezas y debilidades con respecto a la escritura que ha desarrollado hasta este momento de su vida escolar; para complementar lo manifestado por los estudiantes en la encuesta, se diseñaron y aplicaron dos instrumentos, uno dirigido a docentes y otro a los padres de familia de los estudiantes con los cuales se está desarrollando la investigación.

- Exploración o planteamiento de las hipótesis como acciones a realizar para cambiar la práctica. Los resultados obtenidos en el primer momento, se constituyen en la base para construir la propuesta que tendrá como fin dotar a los estudiantes de herramientas que hagan eficiente la producción escrita, susceptible de ser utilizada en las diferentes actividades escolares y sociales que el joven requiera.

- Construcción del plan de acción, comprende la revisión del problema inicial, acciones concretas requeridas, visión de estrategias y planificación de instrumentos, sin olvidar la puesta en marcha del primer paso en la acción, evaluación y revisión del plan general (Bisquerra, 2014, p. 378). Es decir, diseñar e implementar una propuesta pedagógica para fortalecer la habilidad escrita de los estudiantes. Finalmente, construir un compendio de los cuentos elaborados por los estudiantes, como resultado de la práctica de la propuesta y evaluar de qué manera influyó en el desarrollo de la habilidad escrita de los estudiantes.

\section{Resultados}

Teniendo en cuenta lo anterior se realizó una serie de pruebas diagnósticas que incluye a estudiantes, docentes y padres de familia, con el propósito de revelar las debilidades y fortalezas relacionadas con la coherencia, cohesión, uso de reglas ortográficas, vocabulario $\mathrm{y}$ estructura de texto entre otros.

La prueba aplicada a los estudiantes se desarrolló en dos momentos, en el primero el estudiante contesta preguntas relacionadas con la frecuencia con la que desarrolla actividades de escritura en su casa, temas favoritos a la hora de escribir y libros de los que se vale para desarrollar este tipo de actividades; también se quiso indagar si los estudiantes consideran que la escritura es importante en la adquisición de conocimientos, en las distintas circunstancias académicas y cotidianas en las que ellos se desenvuelven y, si consideran que en la institución 
educativa se proveen los espacios para desarrollar actividades de escritura de algún tipo.

$\mathrm{Al}$ analizar esta prueba se pudo observar que los estudiantes consideran que poseen habilidades apenas aceptables para escribir, ya que la realizan de manera esporádica. De otra parte, manifiestan que en algunas oportunidades les llama la atención escribir cuentos especialmente si ellos son de ficción, cabe anotar que los jóvenes están de acuerdo en que las personas que leen y realizan actividades de escritura desarrollan habilidades que les facilitan la adquisición de conocimientos, más que las personas que no lo hacen, ya que lo han experimentado de manera personal. En otras palabras, los estudiantes manifiestan su poco interés por realizar tareas de escritura, siendo conscientes que al intentar hacerlo encuentran dificultades al empezar a escribir porque no saben cómo hacerlo, saben que tienen faltas de ortografía, no saben cómo utilizar los tiempos verbales, carecen del vocabulario suficiente para expresar sus ideas y por tanto lo que escriben en muchas ocasiones no se comprende fácilmente. Para terminar los estudiantes manifiestan que el colegio no provee los espacios que propician la escritura.

En un segundo momento del diagnóstico los estudiantes se enfrentan a la escritura valiéndose de una imagen como base para realizar un cuento. En esta parte de la prueba se obtuvieron los siguientes resultados:

Con respecto a la coherencia del texto elaborado por los estudiantes, es preciso anotar que la coherencia hace relación a que un texto tenga sentido y sea de fácil comprensión. El ejercicio da a conocer que de los 28 estudiantes que conforman este grado, a 21 se les dificultan escribir un texto con las mencionadas características y el resto lo logran, pero en pequeños textos que no pasan de tres o cuatro renglones.

Entre tanto, la cohesión hace relación a la utilización de varios factores como los conectores, (sino también, por el contrario, porque, por lo tanto, entre otros) la utilización de las normas y signos de puntuación, la ortografía y las formas verbales adecuadas, es decir que utilicen los verbos correctamente. Teniendo en cuenta estas características el uso ortográfico en la producción textual, solamente cinco niños utilizan correctamente las normas básicas de ortografía, mientras que los otros 23 no conocen ni poseen dominio de las normas elementales de ortografía.

Asimismo, es importante destacar que el único conector gramatical que utilizan 19 estudiantes es el "y", los demás no ponen en juego conector alguno, pues escribieron su texto de un solo párrafo y de tres a cinco renglones. En lo referente a uso de signos en la producción de textual, seis de los estudiantes utilizan el punto aparte cuando el texto que han escrito tiene dos ó más párrafos, y la gran mayoría (22) no utilizan ningún signo de puntuación, quizá esto se debe a que escribieron textos cortos.

Mientras que las formas verbales hacen referencia a la adecuada utilización de los verbos, es decir, sus respectivas personas, tiempos, modo y número. Donde 18
De otra parte, manifiestan que en algunas oportunidades les llama la atención escribir cuentos especialmente si ellos son de ficción, cabe anotar que los jóvenes están de acuerdo en que las personas que leen y realizan actividades de escritura desarrollan habilidades que les facilitan la adquisición de conocimientos, más que las personas que no lo hacen, ya que lo han experimentado de manera personal. 
estudiantes utilizan correctamente el tiempo y la persona de los verbos más comunes, mientras que el resto de los encuestados (10) no demostraron conocimiento sobre su uso.

Finalmente, el análisis de este instrumento demuestra claramente que aspectos se deben tener en cuenta al momento de diseñar e implementar una estrategia pedagógica, que conlleve a fortalecer la habilidad escrita en estudiantes de primer año del bachillerato.

Por su parte, el instrumento aplicado a los docentes constaba de seis preguntas de las cuales una fue abierta. La encuesta hacía referencia a la dedicación que ellos tienen durante la semana a la producción escrita de los estudiantes, dificultades que han detectado en el proceso, las temáticas de interés, actividades desarrolladas con padres de familia para apoyar los procesos de escritura, se indago sobre el apoyo que proporciona la institución al momento de involucrar a los padres de familia en los procesos de lectura de los niños y finalmente se investigó sobre las estrategias lúdicas utilizadas para desarrollar la lectura.

En cuanto al tiempo semanal que dedican los docentes de la institución a la producción escrita de sus estudiantes se evidencia que cinco de ellos emplean entre 15 minutos y una hora para esta actividad; tres de ellos, un tiempo de 30 minutos y los restantes (2) no le dedican ningún tiempo.

Por lo que se refiere a la dificultad que tienen los estudiantes en la escritura, nueve docentes creen que a los estudiantes se les dificulta hacer textos usando correctamente las reglas de ortografía y así mismo con poca coherencia; dos de ellos han identificado que los estudiantes tienen poco interés para practicar la escritura de textos; simultáneamente se ha detectado que hay estudiantes con mayores dificultades para redactar textos con ortografía, coherencia y no saben cómo empezar a escribir un texto; mientras que dos de ellos al evidenciar la falta de interés en la redacción de textos obligan a los estudiantes para que practiquen la escritura y por consiguiente aumentan la apatía hacia este proceso.

De acuerdo a la experiencia de los docentes, cuatro de ellos expresan que a los estudiantes les gusta escribir sobre las experiencias personales; mientras que a tres de ellos les gusta escribir cuentos producto de su imaginación, a diferencia del restante de los encuestados quienes afirman que lo hacen por tareas escolares.

Por otra parte, las actividades que los docentes realizan con los padres de familia para apoyar la escritura se limitan a los talleres que programa la institución, esto lo expresan tres docentes; al contrario, los otros siete indican que no se realiza ninguna actividad para apoyar el proceso. De aquí que los docentes consideren que el apoyo que proporciona la Institución de involucrar a los padres de familia en el proceso de lectura y escritura en los niños es inexistente, no hay espacios adecuados para esta clase de actividades. 
En el análisis se interpretan las respuestas que dieron los profesores de acuerdo con las asignaturas que orientan. Los docentes de matemáticas utilizan como estrategia el planteamiento de situaciones problemas cotidianos para implementar el uso de la escritura, luego ellos consideran que lo lúdico hace referencia a lo que para los estudiantes sería práctico y útil en su vida diaria; por su parte los profesores que orientan las humanidades se les facilitan más la parte lúdica para la implementación de estrategias en el desarrollo de la lectura por medio de talleres, ensayos, cuentos, tareas, coplas, historietas, actividades de asociación de conceptos y para terminar, los docentes de ciencias sociales, ciencias naturales y filosofía acuden directamente a la lectura y comprensión de textos con la lectoescritura de noticias actuales relacionadas con el tema y elaboración de resúmenes.

A continuación se presenta el análisis de la encuesta aplicada a los padres de familia, conformada por siete preguntas referidas a: tiempo que dedican en apoyar a los hijos en la lectura y escritura, tipo de actividades que desarrollan para fortalecer esos procesos, importancia de la lectura y la escritura en la adquisición del conocimiento, frecuencia con la que esta se practica, aspectos en los que practica la escritura y opinión sobre actividades de producción escrita desarrolladas en la institución para mejorar las habilidades en el aprendizaje de sus hijos.

Para empezar con la dedicación del tiempo de los padres hacia los hijos en lo referente a la lectura y la escritura, 19 de ellos lo hacen cada vez que sus hijos lo necesitan; seis de ellos los apoyan esporádicamente, mientras que un padre de familia solamente una vez por semana y los otros dos no dedican tiempo. Con respecto a la actividad que más realizan en los hogares para fortalecer la lectura y escritura, en 11 hogares se realiza la lectura de la biblia; al contrario de seis hogares que lo hacen elaborando las listas de mercado y los restantes con el diligenciamiento de facturas.

En consideración de la importancia de la lectura y la escritura en la adquisición del conocimiento, 22 padres de familia afirman que siempre será importante; sin embargo, cuatro de ellos manifiestan que casi siempre se adquieren conocimientos por este medio y solo dos opina que no es importante para adquirir conocimiento. La frecuencia con la que los padres de familia practican la lectura y la escritura es: algunas veces 12 de ellos, casi siempre, cinco de ellos; y 11 de ellos, siempre.

En cuanto a la comunicación de los padres de familia con los docentes de sus hijos se evidencia que 13 de ellos solo se comunican cuando lo necesitan, ocho con los directores de curso cuando hay entrega de calificaciones, seis de ellos se reúnen con los docentes cuando son notificados y los demás no respondieron a esta pregunta. Los padres de familia de la Institución practican la escritura en los correos electrónicos y en la creación de textos, así lo manifiestan 16 de ellos; haciendo peticiones a la Institución cuatro de ellos; dos padres 
Ander-Egg (2003) mencionado por Moreno (2015), la triangulación de datos hace referencia a la utilización de diferentes estrategias y fuentes de información sobre una recogida de datos que permite contrastar la información recabada, así como obtener una mayor validez y confiabilidad de dichos datos elaborando cartas; un padre de familia solo cuando es necesario, otro haciendo cuentas, otro marco todas las opciones $\mathrm{y}$, ninguna de las opciones dadas, tres padres de familia.

En definitiva 22 padres de familia consideran que las actividades de producción escrita siempre mejoran la lectura y la escritura en los estudiantes, sin embargo, cinco de ellos plantean un casi siempre y uno de ellos no escribió ninguna respuesta.

En consecuencia, la mayoría de los padres de familia dedican tiempo a sus hijos cuando ellos así lo requieren y promueven actividades de lectura y escritura con la realización de cuestiones cotidianas como la lectura de la biblia, las listas del mercado y diligenciar facturas. De ahí la importancia que ellos otorgan a la lectura y a la escritura en la adquisición de conocimientos en coherencia con la frecuencia con la que realizan ejercicios prácticos de lectura y escritura. Sin embargo, es evidente la falta de comunicación de los padres de familia con los docentes de sus hijos ya que la refieren solo a cuando la necesitan o cuando son notificados y por supuesto cuando hay entrega de calificaciones. Al contrario de lo que refleja la práctica de la escritura que realizan los padres, donde un alto porcentaje redactan correos electrónicos y crean diferentes tipos de textos incluidas las cartas y peticiones particulares a la institución donde estudian sus hijos.

\section{Discusión de Resultados}

La triangulación es una técnica de investigación que consiste en ubicar en un triángulo las diversas miradas o datos de la investigación, para revisarlos desde los tres ángulos, en cada uno de los cuales se presenta una intersección de dos miradas o perspectivas, o también, de dos tipos de datos. Forma parte del proceso de validación en el análisis de la información, lo que significa que los múltiples documentos y miradas se cruzan para contrastar la información y complejizar las dimensiones arrojadas por estos cruces (Cerda, 2011).

Para Ander-Egg (2003) mencionado por Moreno (2015), la triangulación de datos hace referencia a la utilización de diferentes estrategias y fuentes de información sobre una recogida de datos que permite contrastar la información recabada, así como obtener una mayor validez y confiabilidad de dichos datos. Teniendo en cuenta lo anterior, de manera puntual se utilizó una forma de triangulación de personas, es decir, se aplicó un mismo tipo de instrumentos para conocer las fortalezas y las debilidades en la producción escrita en los estudiantes de grado sexto, desde la perspectiva del docente, padre de familia y por supuesto el estudiante, de tal forma que la categoría analizada es la habilidad escrita, como se puede observar en la figura 1.

La triangulación de personas se realiza teniendo en cuenta un análisis colectivo por tratarse de un grupo de estudiantes pertenecientes a una comunidad educativa en la que las interacciones entre padres de familia y docentes influyen en la colectividad total de la unidad de análisis (estudiantes de grado sexto). 
Figura 1.

Triangulación de información Estudiantes

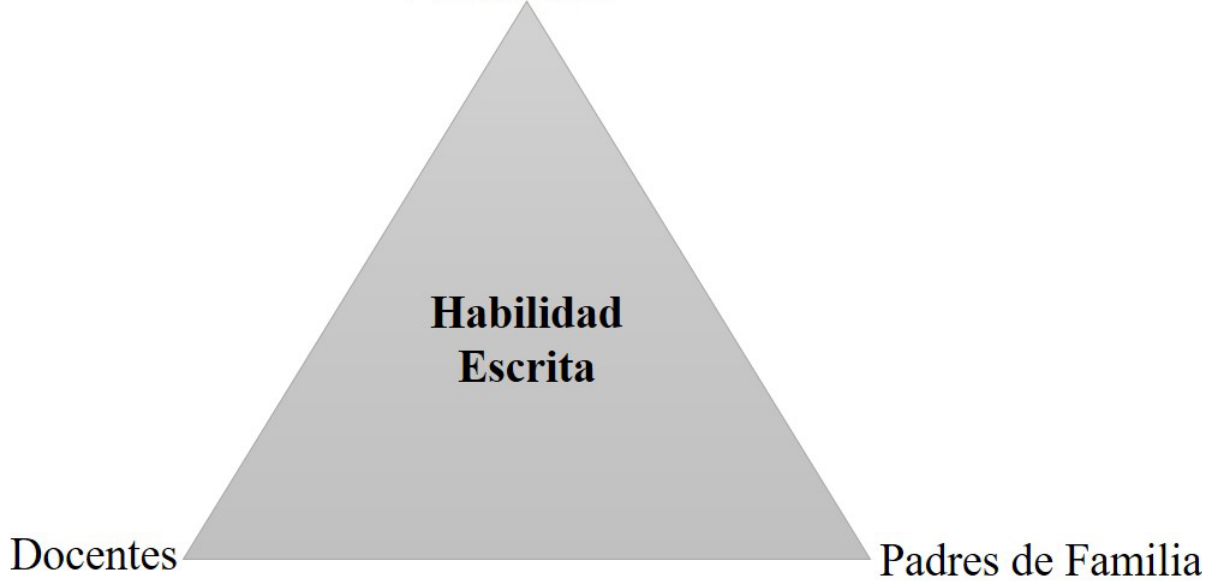

Fuente: Elaboración propia.

\section{Docentes}

Sin lugar a dudas el tiempo que dedican los docentes de la institución a la producción escrita de los estudiantes es un factor determinante en la detección de las dificultades para realizar o hacer textos utilizando correctamente las reglas de ortografía, la coherencia, la cohesión, los tiempos verbales, el uso de conectores y de signos; sin embargo al realizar el análisis de los tiempos que los docentes dedican a esta actividad son insuficientes y por lo tanto se infiere que esta falta de interés por parte de los docentes conlleva a reiterar el problema objeto de estudio que se aborda en esta investigación y por ende incorporar estrategias pedagógicas y didácticas pertinentes a la población objeto de estudio, independientemente de las áreas de conocimiento que orienten los docentes de la Institución; de tal forma que motiven a los estudiantes en la redacción de textos en todos los ámbitos de su vida y no solamente por cumplir con las tareas escolares, como lo afirman los mismos docentes.

\section{Padres de Familia}

La participación de los padres de familia en el apoyo de lectura y escritura que realizan sus hijos en el hogar es determinante porque apoyan las actividades desarrolladas en la institución; no obstante el resultado del instrumento aplicado deja entrever que ellos solo le dedican tiempo a los hijos cuando lo necesitan, de manera esporádica y algunos no lo hacen, a pesar de que consideran que la lectura y la escritura es un factor importante en la adquisición del conocimiento, lo cual es reiterado en la frecuencia con la que ellos practican la lectura y la escritura. En este mismo sentido se evidencia la comunicación de los padres 
con los docentes de sus hijos, ya que en su mayoría manifiestan que lo hacen por necesidad o cuando hay entrega de calificaciones, además ellos consideran que las actividades de lectura y escritura se promueven con actividades como la elaboración de listas de mercado y diligenciamiento de facturas; lo cual no implica necesariamente la redacción de un texto académico con las características que denotan una producción escrita.

\section{Estudiantes}

Contrastando los hallazgos en el análisis de los instrumentos aplicados a los docentes y a los padres de familia, los estudiantes consideran que poseen habilidades apenas aceptables para escribir y que eventualmente les llama la atención escribir cuentos de ficción; sin embargo en el segundo momento de la prueba diagnóstica a la mayoría de ellos se les dificulto escribir un texto valiéndose de una imagen, pues solamente cinco de ellos utilizan correctamente las normas básicas de ortografía, a diferencia del uso de los conectores gramaticales en donde solamente utilizaron el "y", al igual que los signos de puntuación. No obstante 18 estudiantes utilizan correctamente las formas verbales de los verbos más comunes.

En correlación con lo anteriormente expuesto los estudiantes manifiestan que el colegio no provee espacios para propiciar la escritura, luego esto concuerda con lo afirmado por los docentes y la evidente falta de comunicación de los padres de familia con los docentes de sus hijos.

\section{Conclusiones}

La lectura y la escritura son función obligatoria de la escuela, la cual debe conformar comunidad de lectores y escritores, lo que se constituye en un gran reto para las instituciones educativas, ya que deben conformar semilleros académicos con tal fin. Un gran desafío por incorporar a esos jóvenes a la cultura de lo escrito. Para esto es necesario que la escuela oriente a los estudiantes y brinde acceso a los textos de una manera libre, que motiven no solamente a leer sino a practicar la escritura como un medio para expresar sus ideas, inquietudes, sueños y necesidades.

Por tal razón es importante que desde la escuela se propicie la habilidad en producción de textos, ya que ello favorece la adquisición de conocimiento y ayuda a que la organización de la información sea eficiente, se creen formas de planeación sobre lo que se escribe, se mejore la redacción, la ampliación del vocabulario y la creación de hábitos de revisión, reescritura, edición de lo que se está escribiendo y si es necesario la defensa y argumentación de las producciones realizadas.

En este sentido el docente debe promover la escritura en estrecha relación con la cultura a través de la observación en las aulas, de qué y cómo escriben sus estudiantes; averiguar el concepto que tienen de la escritura; diseñar guías de ayuda que faciliten el proceso de escribir; fomentar el hábito de hacer esquemas y resúmenes, promover la elaboración de borradores para inculcar la costumbre de 
revisar los textos, editarlos, exponerlos y defenderlos ante un público.

Entonces, la escritura de cualquier género discursivo interviene no solo la cuestión gramatical o léxica, sino también las convenciones socioculturales de cada disciplina. En otras palabras, el texto depende de las instituciones en las que se producen, de los autores y lectores que los procesan. Por ejemplo, el desarrollo de la habilidad escrita se hace indispensable, puesto que en la vida cotidiana es de mayor utilidad, cuando se requiere redactar un oficio a una institución para reclamar los derechos, o cuando exigen enviar una excusa al colegio porque un hijo no pudo asistir a las actividades académicas.

Como se ha expuesto, es importante intervenir las aulas para mejorar las prácticas de escritura implícitas en los procesos de enseñanza y aprendizaje en la producción de textos, abriendo espacios que favorezcan la reflexión, la interiorización y el dominio de las competencias básicas en la escritura desde los diferentes géneros discursivos; de tal forma que se puedan incorporar diversas estrategias con los estudiantes que motiven el desarrollo de sus actividades escolares en todas las áreas del conocimiento haciendo énfasis en el cuento como recurso didáctico.

\section{Referencias}

Alba, D., \& González, A. (2015). Enseñar a argumentar escribiendo. (Tesis de maestría). Universidad Pedagógica y Tecnológica de Colombia , Tunja.

Álvarez, T., \& Ramírez, S. (2006). Teorías o modelos de producción de texto en la enseñanza y aprendizaje de la escritura. Didáctica (Lengua y literatura), 29-60.

Álvarez, T. (2010). Competencias básicas en escritura . Barcelona: Octaedro.

Barletta, N., Toloza, H., Del Villar, L., Rodríguez, A., \& Bovea, V. (2013). Teaching and learning of reading and writing. A conspiracy in the public context, 133-168.

Bazán, A., Urbina, D., Domínguez, L., \& Mansillas, M. (2011). Desarrollo funcional de competencias de producciòn de textos en alumnos con bajo desempeño en el ùltimo año de primaria. Revista Méxicana de Análisis de Conducta, 37(1), 89-107.

Bettelheim, B. (1988). Psicoanálisis de los cuentos de hadas: la extraordinaria importancia de los cuentos de hadas para la formación moral e intelectual de los niños. . Barcelona: Crítica.

Bronckart, J. (1985). Las ciencias del lenguaje: un desafío para a enseñanza? París : UNESCO. 
Bruner, J. (1987). La importancia de la educación. Barcelona : Paidós.

Contursi, M. \& Ferro, M. (2006). La narración: Usos y teorías. Bogotá : Norma.

Gúzman, K. \& Rojas, L. (2012). Escritura colaborativa en alumnos de primaria: Un modelo social de aprender juntos. Revista mexicana de investigación, 17(52), 217-245.

Habermans, J. (1989). Teorías de la acción comunicativa: Complementos y estudios previos . Madrid : Cátedra.

Henao, O., Ramírez, D. \& Mejía, J. (2009). La producción escrita mediada por un programa que reconoce la voz. Una experiencia con estudiates de sexto grado. Revista iberoaméricana de informática educativa , 50-59.

Hernández, J. (2008). Elaboración de un texto escolar de lengua castellana, con un enfoque discursivo para el grado sexto dentro de un contexto socio-cultural específico. . Tesis de grado. Tunja, Universidad Pedagógica y Tecnológica de Colomia , Tunja: UPTC.

Holliday, M. (2005). El lenguaje como semiótica social . México: Fondo de cultura económica .

Meneses, G. (1955). Antología del cuento venezolano. Caracas: Ministerio de Educación

Orrego, J., \& Toro, L. (2014). Relaciones vitales: el aula como escenario permanente de investigación. Praxis \& Saber, 5(10), 121 - 139. https://doi. org/10.19053/22160159.3025

Ricoeur, P. (2004). El modelo del texto: la acción comunicativa como texto. México : Fondo de cultura económica .

Van Dijk, T. (1983). La ciencia del texto. Barcelona: Paidós.

Vigotski, L. (1985). Pensamiento y lenguaje. Buenos Aires: La Pléyade. 